\title{
Clinical Medicine: Advances in Implantable Cardiac Devices
}

\author{
Jorge Palazollo MD, Sheldon M. Singh MD
}

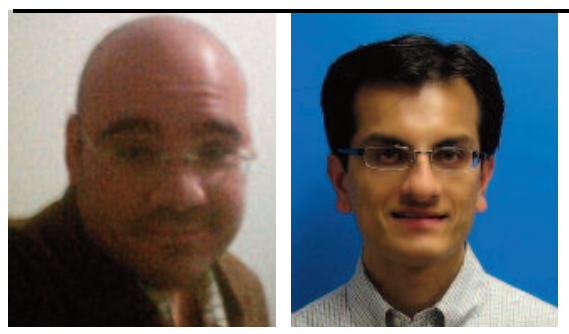

\begin{abstract}
About the Authors
When this article was written, Dr. Palazollo (far left) was a fellow in Cardiac electrophysiology and pacing at the Schulich Heart Program, Sunnybrook Health Sciences Center in, Toronto, Canada. Dr Palazollo completed his medical school, internal medicine and cardiology residency in Mendoza, Argentina. He subsequently completed basic cardiac electrophysiology Training at McMaster University, Hamilton, Ontario. Dr. Palazollo has a clinical interest in the care of patients with implantable cardiac devices. Dr. Sheldon Singh is a staff electrophysiologist and an assistant professor of Medicine in the Schulich Heart Program, Sunnybrook Health Sciences Centre, and is on the Faculty of Medicine at the University of Toronto. Correspondence may be directed to sheldon.singh@sunnybrook.ca.
\end{abstract}

\section{Introduction}

The use of implantable cardiac devices has increased and will continue to increase as the population ages. For more than 50 years, patients with a slow pulse have enjoyed the benefits of cardiac pacing to alleviate symptoms and, in some cases, prolong life. Implantable cardiac devices have evolved; in addition to providing pacing support, some devices - namely, implantable cardioverter defibrillators (ICDs) - can also terminate malignant ventricular arrhythmias, facilitate the synchronization of ventricular function, monitor clinical variables, and store a wealth of information to assist clinical management. This review discusses currently available device diagnostics, highlights some advances in implantable cardiac device therapy, and provides a preview of future technologies that may improve the care of patients who have these devices.

\section{Atrial Fibrillation}

Atrial fibrillation (AF) is the most common sustained arrhythmia worldwide. Patients with AF carry a threefold-tofivefold increased risk of stroke. This risk is present even in patients with asymptomatic or subclinical AF.

Implantable cardiac devices, particularly those devices with a pacing lead within the atrium, can accurately detect atrial activity. Device diagnostics can allow one to make a precise diagnosis of AF, as well as quantify the duration of each episode and overall burden of the arrhythmia (Figure 1A). This information may provide guidance as to the need for oral anticoagulation, an important consideration given the economic impact of AF-related stroke. The Asymptomatic Atrial Fibrillation and Stroke Evaluation in Pacemaker Patients
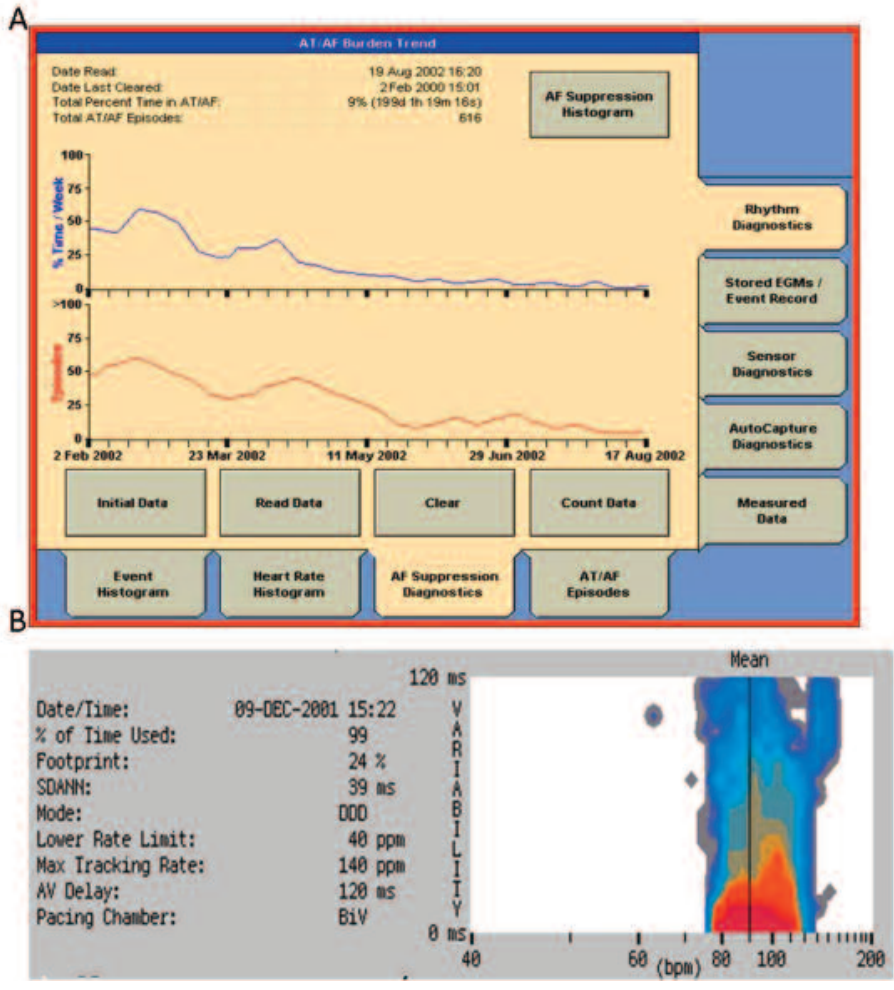

Figure 1. A, Atrial fibrillation burden, including the total time in atrial fibrillation and the number of episodes. B, Heart rate variability for a patient with congestive heart failure. $\mathrm{AF}=$ atrial fibrillation

and the Atrial Fibrillation Reduction Atrial Pacing Trial (ASSERT), which was reported in the New England Journal of Medicine, demonstrated that for patients with subclinical atrial arrhythmias lasting greater than 6 minutes, the odds of an ischemic stroke were 2.49. ${ }^{1}$ 
Although further work is needed to determine whether oral anticoagulation may reduce this stroke risk, the provision of knowledge about the presence of subclinical atrial arrhythmias is an important feature of implantable cardiac devices. Clinicians should be aware of the value of this information, which can direct stroke reduction therapy. In addition to the ability to make decisions on the need for oral anticoagulation, the ability to accurately quantify the patient's AF burden and average heart rate while in AF is also valuable to clinicians and ensures both adequate rate control and an appropriate response to anti-arrhythmic drugs.

\section{Congestive Heart Failure}

Despite advancements in medical therapy for congestive heart failure (CHF), the rates of $\mathrm{CHF}$ admissions have improved little in the past few decades. In the United States, half of all costs for $\mathrm{CHF}$ are related to in-hospital management. Additionally, readmission for $\mathrm{CHF}$ is not trivial; one-quarter of $\mathrm{CHF}$ patients require rehospitalization within 30 days of discharge. Implantable cardiac devices - specifically ICDs - obtain a vast wealth of clinical information that may be useful in the care of CHF patients and potentially reduce the need for hospitalization.

One approach to assessing the development of pulmonary congestion is to measure intrathoracic impedance. Simply stated, when an electrical current is passed across the lung (by pulsing energy from the lead placed in the heart to the pulse generator located in the chest wall), the impedance to this flow of current can be determined. With the development of pulmonary congestion, this impedance will drop, as fluid has better conductance than air. Monitoring day-to-day changes in impedance may allow one to identify the early onset of pulmonary congestion and may - through prompt treatment - avert a hospital admission.

The measurement of heart rate variability is yet another way to assess the severity of CHF and changes in the status of patients with CHF (Figure 1B). Simply stated, heart rate variability is the beat-to-beat variability in consecutive QRS intervals. Worsening heart failure is associated with increased sympathetic activation, higher levels of circulating catecholamines, and a reduction in the normal heart rate variability; these have been correlated with reduced survival. Reduced HR variability is an important indicator for intensification of care in this patient population.

Other physiologic information about progressive CHF is captured by implantable cardiac devices; this includes information on the burden of arrhythmias (both atrial and ventricular) and on elevated nocturnal heart rates. Having this

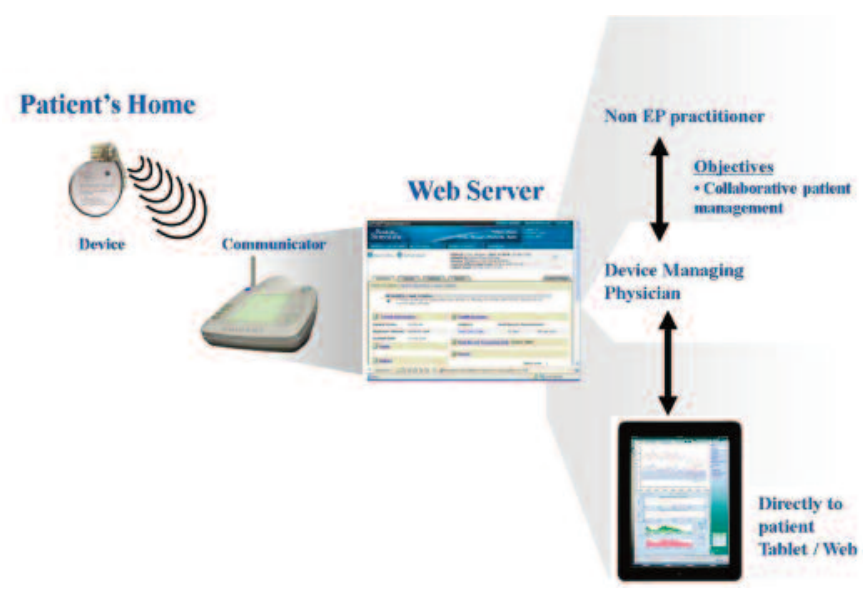

Figure 2. Remote monitoring.

information may allow clinicians to identify patients at higher risk, who might benefit from treatments that will keep them well and out of hospital. ${ }^{2}$

New, more precise hemodynamic assessments with pressure sensors directly monitoring the left atrial and the pulmonary arterial pressure will be available. The CHAMPION study assessed the role of clinician-guided management of CHF patients by direct pulmonary arterial pressure monitoring with the CardioMEMS pulmonary artery pressure monitor. ${ }^{3}$ Results showed a large reduction $(\mathrm{HR}=0.72)$ in hospitalizations of patients with CHF managed with this device. The use of novel and existing tools may have a significant role in improving the care of patients with CHF.

\section{Remote Monitoring}

Accessing the wealth of information available from cardiac devices, particularly for day-to-day monitoring, would be cumbersome if the data could be accessed only as it has been traditionally - that is, with patients visiting their local device clinic. The time and cost associated with this would likely be a deterrent to accessing and utilizing this information for routine clinical use. Device-based algorithms are used in an attempt to overcome this barrier; programmable algorithms are available in implantable devices that inform patients of adverse events by audible alerts or alarms, prompting the patients to seek medical care.

Recently, remote monitoring has become available, bringing patients and caregivers closer (Figure 2). Home bedside transmitters can communicate wirelessly with a patient's implantable device, download information, and transmit that information to a central secure website accessible to caregivers. This approach not only provides some safety regarding device malfunction but can also provide caregivers access to the clinical diagnostics described above, thereby allowing more- 
A

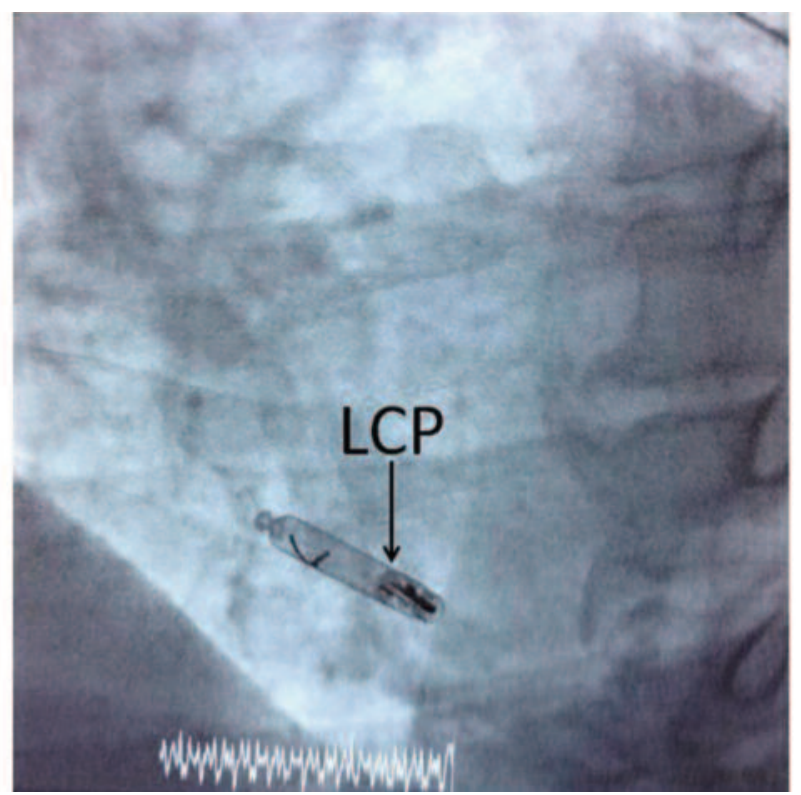

Figure 3. A, Fluoroscopic image of a leadless cardiac pacemaker (LCP).6 www.abstractsonline.com. B courtesy of www.medgadget.com)

tailored patient care. Evidence indicates that this approach does accelerate the identification of new clinical events. Data collection and storage, patient confidentiality, and caregiver responsibilities for acting on the data are currently being addressed at centres that employ this technology. ${ }^{4}$

\section{New Devices}

\section{Magnetic Resonance Imaging Conditional Devices}

Currently, the presence of implantable cardiac devices is considered to be a relative contraindication to magnetic resonance imaging (MRI). This may be problematic, as a large proportion of patients with an implantable device may require MRI during their lifetime. Several concerns arise with MRI of patients with implantable cardiac devices. These include the torque placed on the lead in the magnetic field, which may result in lead dislodgement; pacing behaviours that may change when exposed to a strong magnet; and lead heating, which may injure the adjacent cardiac tissue or actually stimulate the heart. Magnetic resonance imaging-conditional cardiac devices (both pacemakers and defibrillators) are now available. Currently, Health Canada approves MRI-compatible pacemakers for routine use, whereas MRI-compatible ICDs are available under the Special Access Program. These newer devices have less ferromagnetic components which make movement within the MRI field less likely, and have been shown to cause no increased risk in patients undergoing scanning with a 1.5 tesla scanner. Additional data on the safety of scanning with higher-power magnets is necessary, but the general notion is that these devices are likely safe. ${ }^{5}$

\section{B}

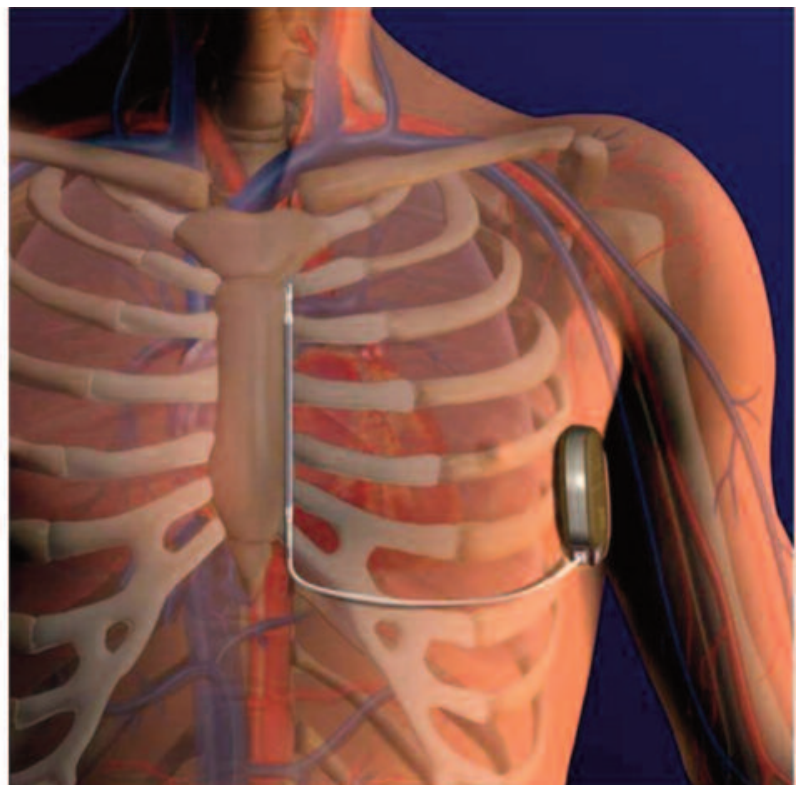

B, A subcutaneous implantable cardioverter defibrillator. (A courtesy of

\section{Leadless Pacemakers}

Leads are an important - if not the most important component of pacing systems, because this portion of the device facilitates cardiac pacing and is used to derive information on clinical events. Unfortunately, leads are considered the weakest link of an implantable cardiac device system, as they are exposed to mechanical stress with cardiac and thoracic wall motion. This can result in lead fracture or a breach of insulation. In addition, the risks associated with acute lead implantation, including the risk of pneumothorax or cardiac perforation, are not trivial. Chronic issues that are associated with lead placement within the vascular system include venous occlusion, thrombus formation, and bacterial seeding (of which the resulting device infection can be catastrophic). Such issues are particularly important for young patients who are receiving implantable devices, which expose these patients to a long-term risk of lead complications and the associated morbidity.

Leadless pacemakers have recently been developed and are currently being evaluated in the clinical arena (Figure $3 \mathrm{~A}){ }^{6}$ Unlike a pulse generator implanted in the chest wall (with accompanying leads placed within the heart), a leadless pacemaker is a self-contained device with battery, diagnostic, and pacing functions. The device is delivered through a large delivery sheath from the femoral vein and screwed into the heart; once positioned, the device remains tethered to the heart. These devices, which are less than $10 \%$ the size of traditional pacemakers, can perform the same functions that are performed by pacemakers and can avoid the need for leads. 


\section{Subcutaneous Defibrillators}

The desire to minimize implantable hardware has also resulted in the development of a defibrillator that does not need transvenous leads. The totally subcutaneous implantable defibrillator is approved by the US Food and Drug Administration and is available through the Health Canada Special Access Programme (Figure 3B). Preliminary studies have shown that this device may sense malignant ventricular arrhythmias in a manner similar to that of a traditional defibrillator and may provide life-saving therapies ${ }^{7}$; however, there are some lingering concerns. Additionally, the absence of any intravascular component has resulted in an inability to provide pacing for those patients who may require pacing capacity in addition to the capability to terminate malignant ventricular arrhythmias.

\section{Conclusion}

Implantable cardiac devices can improve the patient's symptoms and the quality and length of his or her life. Work aiming to improve on device designs that reduce device-related complications is ongoing. Furthermore, advances in software and device algorithms to detect subclinical arrhythmia and the monitoring of other related conditions will provide even better clinical outcomes for patients with implanted cardiac devices.

\section{References}

1. Healy JS, Connolly SJ, Gold MR, et al. Subclinical atrial fibrillation and the risk of stroke. N Engl J Med 2012;366:120-9.

2. Cowie MR, Sarkar S, Koehler J, et al. Development and validation of an integrated diagnostic algorithm derived from parameters monitored in implantable devices for identifying patients at risk for heart failure hospitalization in an ambulatory setting. Eur Heart J 2013;34:2472-80.

3. Abraham WT, Adamson PB, Bourge RC, et al. Wireless pulmonary artery hemodynamic monitoring in chronic heart failure: a randomized controlled trial. Lancet 2011;377:658-66.

4. Yee R, Verma A, Beardsall M, et al. Canadian Cardiovascular Society/Canadian Heart Rhythm Society Joint Position Statement on the use of remote monitoring for cardiovascular implantable electronic device follow-up. Can J Cardiol 2013;29:644-51.

5. Santini L, Forleo GB, Santini M, et al. Evaluating MRI-compatible pacemakers: patient data now paves the way to widespread clinical application? Pacing Clin Electrophysiol 2013;36:270-8.

6. Reddy V, Khairkhahan A, Ligon D, et al. Percutaneous in vivo placement of a novel leadless cardiac pacer: a first-in-man report. Heart Rhythm Society, 2013;

www.abstractsonline.com/Plan/ViewAbstract.aspx?mID=3068\&sKey=ac2b c685-d75f-4de0-af4d-9d6ee388bc51\&cKey=7451777f-88b6-4ec4-a6d0efb6f61dd393\&mKey $=\{$ CA11C8B6-ED27-4A20-953E-64F913C9A29D $\}$ Accessed December 1, 2013.

7. Weiss R, Knight BP, Gold MR, et al. Safety and efficacy of a totally subcutaneous implantable-cardioverted defibrillator. Circulation 2013;128:944-53. 